\title{
Mass movement susceptibility mapping - A comparison of logistic regression and Weight of evidence methods in Taounate-Ain Aicha region (Central Rif, Morocco)
}

\author{
JEMMAH A I ${ }^{1}$ ET AIT BRAHIM L ${ }^{1}$ \\ ${ }^{1}$ Université Mohammed V, Faculté des sciences Département sciences de la terre, Unité de recherche GEORISK et LGRN, 4 \\ Avenue Ibn Battouta Rabat-Agdal, BP 1014, Rabat, Maroc \\ laitbrahim@gmail.com
}

\begin{abstract}
Taounate region is known by a high density of mass movements which cause several human and economic losses. The goal of this paper is to assess the landslide susceptibility of Taounate using the Weight of Evidence method (WofE) and the Logistic Regression method (LR). Seven conditioning factors were used in this study: lithology, fault, drainage, slope, elevation, exposure and land use. Over the years, this site and its surroundings have experienced repeated landslides. For this reason, landslide susceptibility mapping is mandatory for risk prevention and land-use management. In this study, we have focused on recent large-scale mass movements. Finally, the ROC curves were established to evaluate the degree of fit of the model and to choose the best landslide susceptibility zonation. A total mass movements location were detected; $50 \%$ were randomly selected as input data for the entire process using the Spatial Data Model (SDM) and the remaining locations were used for validation purposes. The obtained WofE's landslide susceptibility map shows that high to very high susceptibility zones contain $62 \%$ of the total of inventoried landslides, while the same zones contain only $47 \%$ of landslides in the map obtained by the LR method. This landslide susceptibility map obtained is a major contribution to various urban and regional development plans under the Taounate Region National Development Program.
\end{abstract}

\section{Introduction}

La susceptibilité aux mouvements de mass est une composante importante dans l'équation de ce type de risque, celui-ci étant défini comme la probabilité d'occurrence du phénomène dans un certain secteur.

L'évaluation de celui-ci suppose l'appréciation quantitative et qualitative de la classification, du volume, de la superficie et de la distribution spatiale des mouvements de mass qui existent et/ou qui peuvent apparaître $[1,8]$.

La cartographie de la susceptibilité aux glissements de terrain met en évidence la distribution spatiale des versants potentiellement instables, fondée sur l'analyse des paramètres favorables aux mouvements de mass, par la considération de la superposition spatio-temporelle des facteurs qui ont généré de tels processus dans le passé [2]. A partir du principe «le passé et le présent représentent la clé de l'avenir », on peut formuler les hypothèses sur lesquelles est fondée l'estimation des risques de glissements de terrain :

- la distribution actuelle des glissements reflète les conditions géologiques, géomorphologiques, hydrogéologiques, climatiques, le degré de recouvrement de la végétation, l'impact des activités humaines ;

- les futurs glissements de terrain vont apparaître dans les mêmes conditions ;
- les facteurs de contrôle des glissements peuvent être identifiés, classés, hiérarchisés et évalués par différentes méthodes.

Le degré de fiabilité des cartes de susceptibilité aux glissements de terrain est influencé par plusieurs facteurs, comme la précision, la quantité et la qualité des données acquises, l'échelle de travail, la méthodologie et la modélisation.

Cet article propose ainsi une cartographie de la susceptibilité aux mouvements de mass dans la région de Taounate _ Ain Aicha, prenant en considération des différents aspects et limites méthodologiques. Après une brève présentation du secteur d'étude, la démarche méthodologique ainsi que les résultats seront présentés et discutés.

\section{Contexte de l'étude}

La présente étude s'inscrit dans le cadre d'une étude environnementale portant sur la région de Taounate Ain Aicha. Notre étude vise principalement à développer une approche méthodologique menant à l'élaboration d'une carte de susceptibilité aux mouvements de mass, en appliquant les méthodes de la théorie de l'évidence et la régression logistique et un système d'information géographique (SIG). L'un des objectifs de notre étude est donc de fournir une cartographie simple mais détaillée des zones sensibles aux mouvements de mass. Par ailleurs, cette cartographie peut servir de document de référence aux municipalités régionales pour l'identification des zones 
à risques naturels, lesquelles zones doivent s'inscrire dans les schémas d'aménagement du territoire. Enfin, la région de Taounate _ Ain Aicha a été choisie pour cette étude puisqu'elle constitue l'une des régions du Rif et qu'elles présentent des zones à risques élevés de mouvements de mass.

\section{Zone d'étude et méthodologie}

\subsection{Zone d'étude}

Le secteur étudié forme un rectangle de $640 \mathrm{~km}^{2}$ de surface découpé selon le carroyage rectangulaire de la carte géologique (1/50.000) (Fig. 1).

La région de Taounate _ Ain Aicha au sein du Rif central est caractérisée par une alternance de reliefs montagneux et de vallées dont la plus importante est celle de l'Oued Ouerrhe dont le lit présente par endroits d'importants replats où se développent une agriculture d'irrigation. Cette topographie très contrastée est le résultat des déformations tectoniques de la région au cours du Tortono-messinien et du plio-quaternaire [1, 12] qui a porté en relief des rides de roches résistantes de calcaires et de grès qui sont bordées sur les versants par les roches tendres à moyennement résistantes de marnes, marno-calcaires et schisto-gréseuses. Les constructions, en général se situent sur les crêtes calcaires saturées. Ceci oblige l'expansion anarchique sur les versants argileux nord et sud inaptes à la construction (en plus de mouvements de terrain) sauf

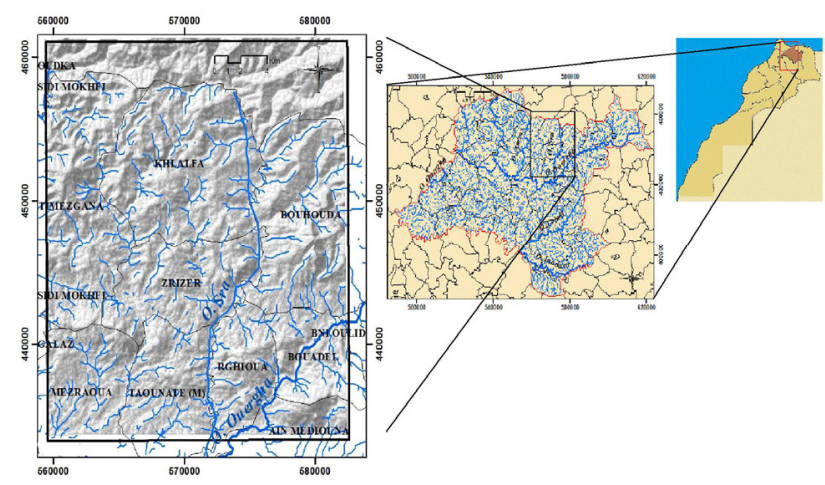

aux abords de l'Ouerrha et de ses affluents.

Fig.1 Situation géographique de la zone d'étude.

\subsection{Méthodologie \\ 2.2.1 Inventaire de mouvements de mass}

L'inventaire des mouvements de mass été conduit via l'utilisation de la base de données existante, complétée la photo-interprétation systématique de l'ensemble du terrain d'étude selon une grille d'analyse. Ceci permet [1] d'éliminer les doublons et erreurs cartographiques de la base de données existante, [2] d'obtenir une représentation de glissements sous forme de polygones séparant la zone d'ablation de la zone d'accumulation pour chacun des phénomènes (fig.2). Quelques campagnes de validation sur le terrain ont permis de valider la qualité de l'inventaire.

La base de données contient finalement 463 phénomènes cartographiés. 339 d'entre eux sont des Eboulis (73\%) et 124 sont des Solifluxion (27\%). Pour le calcul juste $50 \%$ des mouvements de mass sont utilisés pour la modélisation de la susceptibilité. Alors que les $50 \%$ restantes pour la vérification.

\subsubsection{Constitution de la base de données des facteurs de prédisposition}

Les bases de données (cartes géologiques, cartes topographiques, MNT et image satellitaire) ont été utilisées pour dériver les principaux facteurs de prédisposition (lithologie, réseau hydrographique, fracturation, pente, exposition, hypsométrie et occupation du sol,). L'ensemble des données thématiques a été converti en format raster.

- La lithologie représente un facteur conditionnant majeur dans la stabilité des versants aussi bien par, la nature des matériaux (qualité géotechnique des roches) que par la structure (direction et pendage des couches, plans de schistosité, etc.) (fig.3).

- La pente est l'un des facteurs déterminant dans la genèse des glissements de terrain. En effet, pour qu'une rupture puisse se produire sur un versant, il faut qu'il y ait l'intervention de la gravité accompagnée d'un un appel au vide. Ce phénomène ne pouvant prendre naissance sur des terrains plats, la prise en compte du degré de pente des versants et des couches est d'une importance capitale. (fig.4)

- L'hypsométrie, par sa composante altimétrique représente aussi l'un des facteurs les plus déterminants dans la genèse des glissements de terrains. Ainsi, plus l'altitude augmente, plus le risque d'occurrence de mouvement de mass est présent. (fig.5)

- La carte d'exposition (fig.6) à été générée à partir du MNT. Elle Exprime l'orientation des versants par rapport au nord. Elle a été déterminée par mesure, dans le sens des aiguilles d'une montre, de l'angle entre le nord et la projection horizontale de la ligne de pente maximale d'un versant. En se basant sur la connaissance de notre secteur d'étude, nous avons élaborée la carte en cinq classes selon les différentes directions (Plat, N, E, S, et W). Le changement de l'exposition des versants équivaut une grande variabilité de l'obliquité par rapport aux rayons solaire, la durée d'ensoleillement des versants, ainsi que des apports énergétiques (vents, température...) inégaux. Cela rend très différent la prédisposition des versants aux mouvements de mass. Les versants exposés vers le $\mathrm{N}$ et l'W montrent les fréquences les plus importantes d'instabilités de terrain.

- Le réseau hydrographique est généralement liée au phénomène d'érosion hydrique parmi lesquels on retrouve les sapements de berges. Ce phénomène se développe généralement dans les portions des cours 
d'eau où la vitesse d'écoulement est suffisamment puissante pour éroder la partie basale d'un versant. Dans la région de Taounate Ce sont essentiellement les berges des cours d'eau supérieur allant du 3ème au 5ème ordre (selon la hiérarchisation de Strahler), qui sont les plus propices aux instabilités. (fig.7)

- La fracturation peut conditionner ou déclencher un mouvement de masse au niveau de la zone de broyage des failles [3] (cisaillements et/ou décrochements) formée de matériaux fragilisés et permettre aussi (fig.8) l'infiltration des eaux en profondeur qui en contact avec les argiles une fois saturées vont déclencher des instabilités de terrain.

-L'occupation des sols (fig.9) : la cartographie a été réalisée par interprétation des images satellites couplée

aux photographies aériennes (classification multispectrale supervisée) et la base de données de la couverture végétale et surtout des essences forestières mises à notre disposition par le HCEFLCD (HautCommissariat des Eaux et Forêts et de la Lutte contre la Désertification). Cette dernière couche exerce au niveau de la région de Taounate essentiellement une action phytostabilisante sur les versants à substrat argileux ou à substrat rocheux

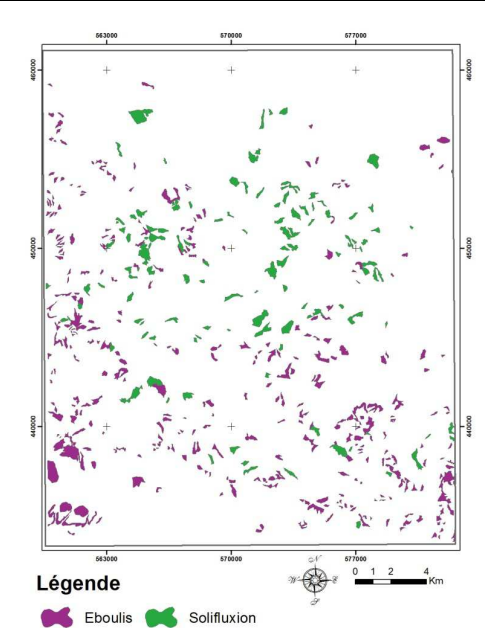

Fig.2 carte d'inventaire des mouvements de terrain

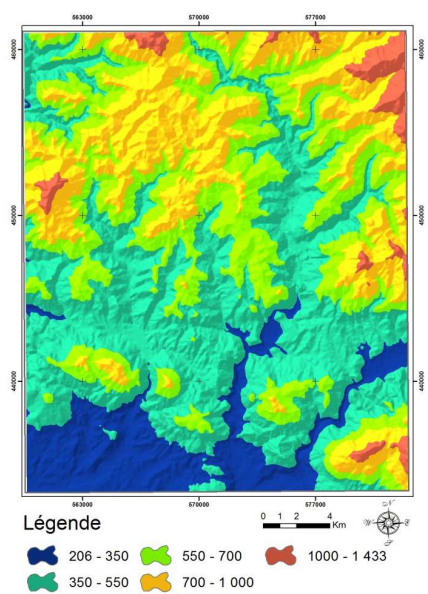

Fig.5 Carte d'hypsométrie (en mètre).

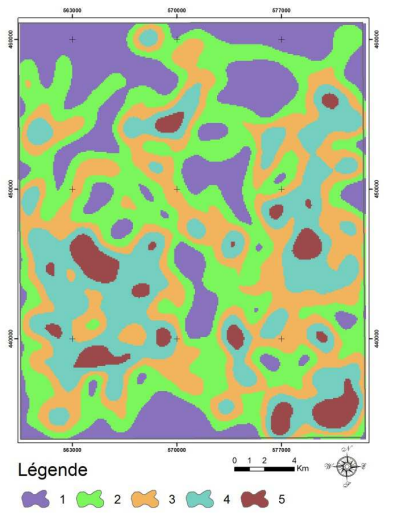

Fig.8 Carte de la densité de la fracturation.

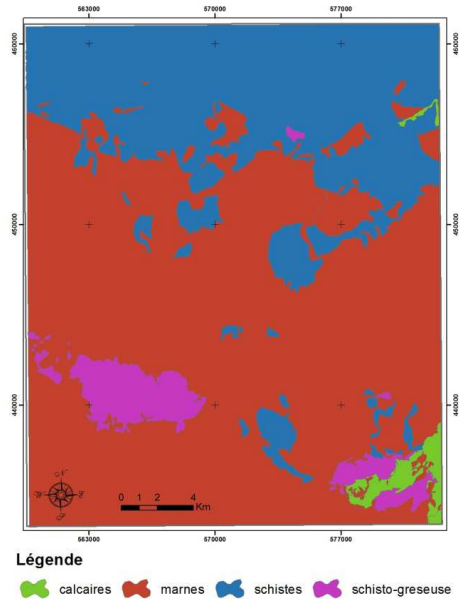

Fig.3 Carte des différentes formations lithologiques.

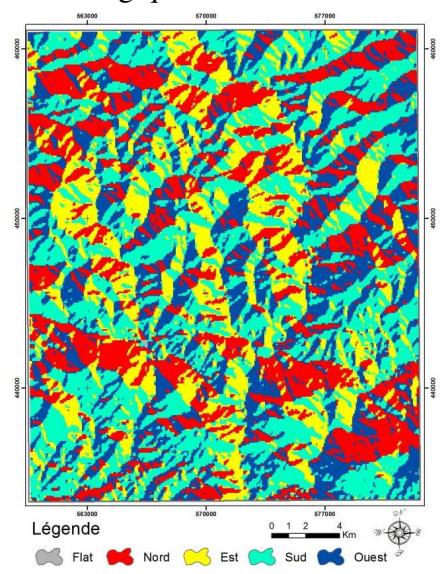

Fig.6 Carte d'expositions.

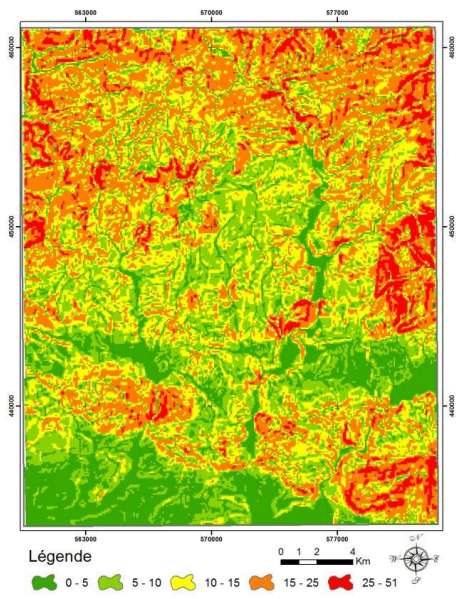

Fig.4 Carte des gradients de pente.

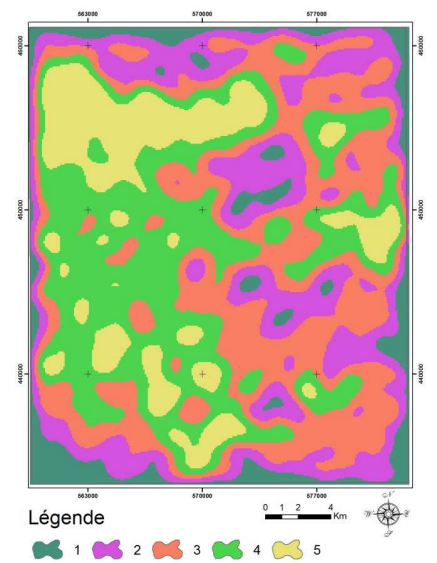

Fig.7 Carte de la densité du réseau hydrographique

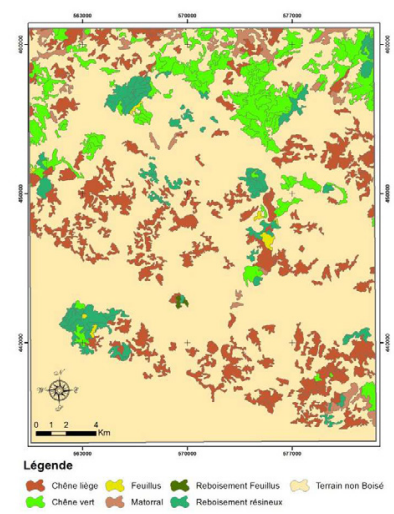

Fig.9 Carte d'occupation du sol 
développant une couverture d'altération argileuse peu épaisse

\subsubsection{La théorie de l'évidence et la Régression Logistique}

Le concept fondamental de ces modèles est d'étudier le comportement d'une variable dépendante (localisation des mouvements de mass) à partir d'une combinaison de variables indépendantes prédictives pour des unités géomorphologiques homogènes.

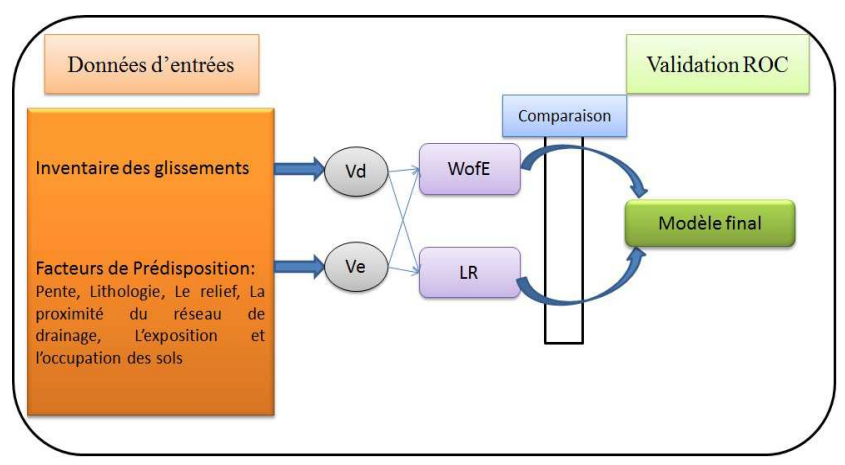

Fig.10 Organigramme de la démarche adoptée à mésoéchelle (1/50 000ème) par la théorie de l'évidence (TDE) et la Régression Logistique

\subsubsection{1 la théorie de l'évidence}

L'analyse spatiale bivariée par la théorie de l'évidence, déjà appliquée en médecine $[4,8,9,10]$ et en géologie [4], est une version log-linéaire du théorème général de Bayes utilisant les principes de calcul de probabilité fondés sur les notions de probabilité a priori et de probabilité a posteriori. La probabilité a priori, qui est la probabilité qu'une unité de terrain (un pixel) contienne une variable dépendante (Vd, dans notre cas les glissements de terrain), est calculée suivant la densité sur la zone d'étude. La probabilité a posteriori est estimée après le calcul de la probabilité a priori selon la densité de Vd pour chaque variable considérée comme prédictive ( $\mathrm{Vp}$, dans notre cas chaque facteur de prédisposition). La méthode est fondée sur le calcul d'un poids positif $(\mathrm{W}+;(1))$ et d'un poids négatif (W- ; (2)), dont les valeurs dépendent de l'association entre $\mathrm{Vp}$ et $\mathrm{Vd}$. [5; 9, 10,11]

$$
W_{+}=\ln \frac{P(B \mid V d)}{P(B \mid \overline{V d})}
$$$$
\text { (2) } W_{-}=\ln \frac{P(\bar{B} \mid V d)}{P(\bar{B} \mid \overline{V d})}
$$

Dans les équations (1) et (2), B est la classe de Vp et le symbole " _ » représente l'absence de Vp et/ou de Vd. Le ratio représente la probabilité de présence sur la probabilité d'absence d'un phénomène. Les pondérations sont additionnées en utilisant le logarithme naturel des ratios appelé logit. Les calculs des valeurs de $\mathrm{W}+$ et de $\mathrm{W}$ - pour toutes les $\mathrm{Vp}$ permettent de calculer la probabilité a posteriori, qui actualise la probabilité a priori. Lorsque plusieurs Vp sont combinées, les zones qui ont un poids respectivement élevé ou faible correspondent respectivement à une plus grande ou une plus petite probabilité de présence de Vd.

\subsubsection{La régression Logistique}

L'analyse spatiale bivariée par régression logistique est un modèle non-linéaire, fondée sur une fonction (3) de répartition sigmoïdale entre la probabilité de présence d'un glissement de terrain $(\mathrm{Vd})$ et différents facteurs de prédisposition $(\mathrm{Vd}),[4,8]$. Le modèle logistique pour la présence ou l'absence de glissements de terrain est défini par :

$$
\operatorname{Pr}(V d)=\frac{1}{1+e^{-z}}
$$

où $\operatorname{Pr}(\mathrm{Vd})$ est la probabilité que la variable dépendante soit présente et $\mathrm{Z}$ est une combinaison linéaire de $\mathrm{Vp}$. Comme $\mathrm{Z}$ varie de $-\infty$ à $+\infty$, le codage de la probabilité varie de 1 à 0 , et la probabilité de présence de $\mathrm{Vd}$ correspond à son espérance conditionnelle. Comme pour l'analyse bivariée par théorie de l'évidence, lorsque plusieurs Vp sont combinées, les zones qui ont un poids respectivement élevé ou faible correspondent respectivement à une plus grande ou une plus petite probabilité de présence de $\mathrm{Vd}$. [8, 9, 10]

\subsubsection{Calage et validation des modèles}

Le calage des modèles a été effectué en ne retenant initialement que $50 \%$ de la population totale de $\mathrm{Vd}$ (présence/absence des mouvements de masse) pour la meilleure combinaison de $\mathrm{Vp}$. Les résultats sont analysés à partir d'une courbe prédictive représentant l'aire cumulée en fonction de la probabilité, sur laquelle des sauts permettent d'identifier trois classes de susceptibilité (faible, moyenne, forte).

La validation des modèles s'effectue d'un point de vue statistique et d'un point de vue expert. Pour la validation statistique, la même procédure que pour le calage est utilisée, en introduisant les $50 \%$ restants de la population totale de Vd. Pour la validation experte, les résultats des modèles en termes de surfaces de glissements de terrain modélisés sont comparés à l'inventaire géomorphologique. [ 9, 10,11]

\section{RESULTATS ET DISCUSSION}

Pour la théorie de l'évidence, les cartes des paramètres de prédisposition combinées avec la carte de mouvements de mass permettent de calculer les poids positifs et négatifs et les contrastes, comme indiqué dans le tableau 1. 
Tableau 1: Corrélation spatiale entre les variables prédictives et les valeurs de pondération calculées par la théorie de l'évidence, $(\mathrm{W}+)$ : la valeur la probabilité a priori, (W-) : la valeur de la probabilité à posteriori, (C) : le contraste qui est la différence entre $\mathrm{W}+$ et $\mathrm{W}$-, $\mathrm{S}(\mathrm{C})$ : la variance du contraste

\begin{tabular}{|c|c|c|c|c|c|}
\hline $\begin{array}{c}\text { Formatio } \\
\text { ns } \\
\text { géologiqu } \\
\text { es }\end{array}$ & $\begin{array}{l}\text { Surface en } \\
\qquad \mathrm{Km}^{2}\end{array}$ & $W+$ & $W$ - & $\begin{array}{c}\text { C(Contr } \\
\text { aste })\end{array}$ & $S(C)$ \\
\hline calcaires & 8,7663 & 0,9677 & $-0,0218$ & 0,9895 & 0,3770 \\
\hline $\begin{array}{l}\text { Schisto- } \\
\text { gréseux }\end{array}$ & 8,0880 & 0,9095 & $-0,0183$ & 0,9278 & 0,4012 \\
\hline Marne & 403,51 & 0,2858 & $-0,8223$ & 1,1081 & 0,1793 \\
\hline Schistes & 213,57 & $-1,2542$ & 0,3207 & $-1,5749$ & 0,2213 \\
\hline $\begin{array}{c}\text { Distance } \\
\text { par } \\
\text { rapport } \\
\text { aux } \\
\text { Failles } \\
\end{array}$ & $\begin{array}{l}\text { Surface en } \\
\qquad \mathrm{Km}^{2}\end{array}$ & $W+$ & $W-$ & $\begin{array}{c}\text { C(Contr } \\
\text { aste })\end{array}$ & $S(C)$ \\
\hline$>500$ & 135,26 & $-0,7003$ & 0,1300 & $-0,8304$ & 0,2139 \\
\hline 400 & 183,34 & $-0,1318$ & 0,0489 & $-0,1807$ & 0,1533 \\
\hline 300 & 154,93 & 0,1595 & $-0,0566$ & 0,2162 & 0,1484 \\
\hline 200 & 133,25 & 0,2349 & $-0,0716$ & 0,3065 & 0,1523 \\
\hline 100 & 30,750 & 0,7087 & $-0,0516$ & 0,7603 & 0,2321 \\
\hline $\begin{array}{l}\text { Gradient } \\
\text { de pente }\end{array}$ & $\begin{array}{c}\text { Surface en } \\
\mathrm{Km}^{2}\end{array}$ & $W+$ & $W$ - & $\begin{array}{c}\text { C(Contr } \\
\text { aste })\end{array}$ & $S(C)$ \\
\hline $0-5^{\circ}$ & 94,264 & $-0,8657$ & 0,0995 & $-0,9652$ & 0,2693 \\
\hline $5-10^{\circ}$ & 151,37 & $-0,0381$ & 0,0118 & $-0,0498$ & 0,1581 \\
\hline $10-15^{\circ}$ & 160,30 & 0,1873 & $-0,0722$ & 0,2595 & 0,1454 \\
\hline $15-25^{\circ}$ & 181,99 & 0,1119 & $-0,0490$ & 0,1610 & 0,1431 \\
\hline $25-51^{\circ}$ & 41,676 & 0,1609 & $-0,0124$ & 0,1733 & 0,2508 \\
\hline $\begin{array}{c}\text { Expositio } \\
n \text { des } \\
\text { versants }\end{array}$ & $\begin{array}{c}\text { Surface en } \\
\mathrm{Km}^{2}\end{array}$ & $W+$ & $W$ - & $\begin{array}{c}\text { C(Contr } \\
\text { aste })\end{array}$ & $S(C)$ \\
\hline Nord & 130,49 & 0,2088 & $-0,0620$ & 0,2708 & 0,1540 \\
\hline Est & 138,03 & $-0,2025$ & 0,0506 & $-0,2531$ & 0,1732 \\
\hline Sud & 187,95 & $-0,1704$ & 0,0651 & $-0,2355$ & 0,1532 \\
\hline Ouest & 171,89 & 0,1434 & $-0,0592$ & 0,2025 & 0,1442 \\
\hline $\begin{array}{l}\text { Distance } \\
\text { au réseau } \\
\text { hydrogra } \\
\text { phique }\end{array}$ & $\begin{array}{c}\text { Surface en } \\
\qquad \mathrm{Km}^{2}\end{array}$ & $W+$ & $W$ - & $\begin{array}{c}\text { C(Contr } \\
\text { aste })\end{array}$ & $S(C)$ \\
\hline$>500$ & 48,199 & $-0,6885$ & 0,0406 & $-0,7291$ & 0,3433 \\
\hline 400 & 128,99 & $-0,3359$ & 0,0706 & $-0,4066$ & 0,1882 \\
\hline 300 & 190,2162 & 0,0941 & $-0,0426$ & 0,1367 & 0,1425 \\
\hline 200 & 180,1351 & 0,2681 & $-0,1267$ & 0,3947 & 0,1390 \\
\hline 100 & 90,8756 & $-0,1390$ & 0,0214 & $-0,1604$ & 0,2018 \\
\hline
\end{tabular}

\begin{tabular}{|c|c|c|c|c|c|}
\hline $\begin{array}{l}\text { Occupatio } \\
\text { n des sols }\end{array}$ & $\begin{array}{c}\text { Surface en } \\
\mathrm{Km}^{2}\end{array}$ & $W+$ & $W$ - & $\begin{array}{c}\text { C(Contr } \\
\text { aste })\end{array}$ & $S(C)$ \\
\hline $\begin{array}{l}\text { Chêne } \\
\text { liège }\end{array}$ & 82,1165 & 0,4044 & $-0,0746$ & 0,4790 & 0,1717 \\
\hline $\begin{array}{l}\text { Chêne } \\
\text { vert }\end{array}$ & 51,6982 & $-0,5528$ & 0,0373 & $-0,5900$ & 0,3124 \\
\hline Feuillus & 1,4756 & & & & \\
\hline $\begin{array}{c}\text { Reboise } \\
\text { ment } \\
\text { Feuillus }\end{array}$ & 24,3778 & $-0,2454$ & 0,0087 & $-0,2540$ & 0,3895 \\
\hline Matorral & 11,6754 & 0,7738 & 0,0102 & $-0,7840$ & 0,7164 \\
\hline $\begin{array}{c}\text { Terrain } \\
\text { non } \\
\text { Boisé }\end{array}$ & 468,6057 & $-0,0099$ & 0,0265 & $-0,0364$ & 0,1496 \\
\hline $\begin{array}{l}\text { 'hypsomét } \\
\text { rie }\end{array}$ & $\begin{array}{l}\text { Surface en } \\
\qquad \mathrm{Km}^{2}\end{array}$ & $W+$ & $W-$ & $\begin{array}{c}\text { C(Contr } \\
\text { aste })\end{array}$ & $S(C)$ \\
\hline $\begin{array}{l}206- \\
400 \mathrm{~m}\end{array}$ & 130,5278 & $-0,1952$ & 0,0457 & $-0,2409$ & 0,1766 \\
\hline $\begin{array}{l}400- \\
600 \mathrm{~m} \\
\end{array}$ & 247,4623 & 0,3252 & $-0,2787$ & 0,6039 & 0,1340 \\
\hline $\begin{array}{l}600- \\
800 \mathrm{~m}\end{array}$ & 167,3002 & $-0,2848$ & 0,0869 & $-0,3717$ & 0,1658 \\
\hline $\begin{array}{c}800- \\
1000 \mathrm{~m}\end{array}$ & 64,8983 & $-0,1894$ & 0,0198 & $-0,2092$ & 0,2376 \\
\hline $\begin{array}{l}1000- \\
1433 \mathrm{~m}\end{array}$ & 19,4222 & $-2,0049$ & 0,0281 & $-2,0330$ & 1,0048 \\
\hline
\end{tabular}

\subsection{Calcul de l'indépendance conditionnel} (IC)

\subsubsection{Degré de liberté entre chaque paire de} variables de predisposition

Parmi les sept variables de prédisposition traités, six ont été retenues (occupation des sols, la pente, le facies, l'exposition, distance aux failles et le réseau hydrographique) étant donné que les valeurs du degré de liberté $(\alpha)$ entre chaque paire de variables obtenue par la méthode de WofE (tableau.2) sont supérieurs à $0,05(\alpha>0,05)$. La variable hypsométrie a été retirée $(\alpha<$ $0,05)$ étant donné sa dépendance avec tous les variables. Ce résultat a été confirmé par le test de chicarrée $\left(\mathrm{X}^{2}\right)$ étant donné que l'écart n'est pas important entre l'écart de X2 observé et X2 théorique pour chaque couple de facteurs et pour l'ensemble des variables à modéliser. $[4,9,10]$

Tableau 2 : Valeurs du degré de liberté $\alpha$ entre chaque paire de variables: (OS,occupation du sol ;Lt,lithologie ; Rh,réseau hydrographique ;Exp, exposition; DF distance des failles)

\begin{tabular}{|c|c|c|c|c|c|}
\cline { 2 - 6 } \multicolumn{1}{c|}{} & OS & Lt & R H & Exp & DF \\
\hline Pente & 0,8969 & 0,6467 & 0,4972 & 0,9999 & 0,7373 \\
\hline OS & & 0,8172 & 0,9999 & 1,0000 & 1,0000 \\
\hline Lt & & & 0,0000 & 0,9948 & 0,0000 \\
\hline RH & & & & 0,9721 & 0,9285 \\
\hline Exp & & & & & 0,7736 \\
\hline
\end{tabular}


L'analyse bivariée par régression logistique est plus souple à mettre en oeuvre car elle ne suppose pas une hypothèse d'indépendance conditionnelle [7, 10], ce qui peut réduire considérablement le temps d'analyse en amont.

D'après les valeurs des coefficients de régression logistique qu'on a réalisée à partir des paramètres de prédisposition (occupation des sols, la pente, le facies, exposition, distance aux failles et le réseau hydrographique), on peut dire que la pente et le réseau hydrographique sont les paramètres les plus importants dans l'apparition des mouvements de mass. $[8,9,10]$

Tableau3: Les valeurs des coefficients de régression obtenus pour les paramètres indépendants

\begin{tabular}{|c|r|r|}
\hline Paramètres & Coefficients & $\begin{array}{c}\text { Standard } \\
\text { Déviation }\end{array}$ \\
\hline Constant & $-2,242407$ & 0,519553 \\
\hline Pente & 0,175012 & 0,060177 \\
\hline Occupation des sols & $-0,014301$ & 0,035736 \\
\hline Lithologie & $-0,772629$ & 0,105654 \\
\hline $\begin{array}{c}\text { Densité du réseau } \\
\text { hydrographique }\end{array}$ & 0,175952 & 0,064487 \\
\hline Exposition & $-0,026988$ & 0,060661 \\
\hline Densité aux failles & 0,142481 & 0,061142 \\
\hline
\end{tabular}

Viens par la suite la densité aux failles, alors que l'occupation des sols, la lithologie et l'exposition avec ce coefficients négatif ne présente pas une grande importance dans l'apparition des mouvements de mass. La carte de susceptibilité aux mouvements de mass la région de Taounate_Ain Aicha permet de distinguer 5 classes de susceptibilité : très forte, forte, moyenne, faible, et très faible (Fig.11et 12).

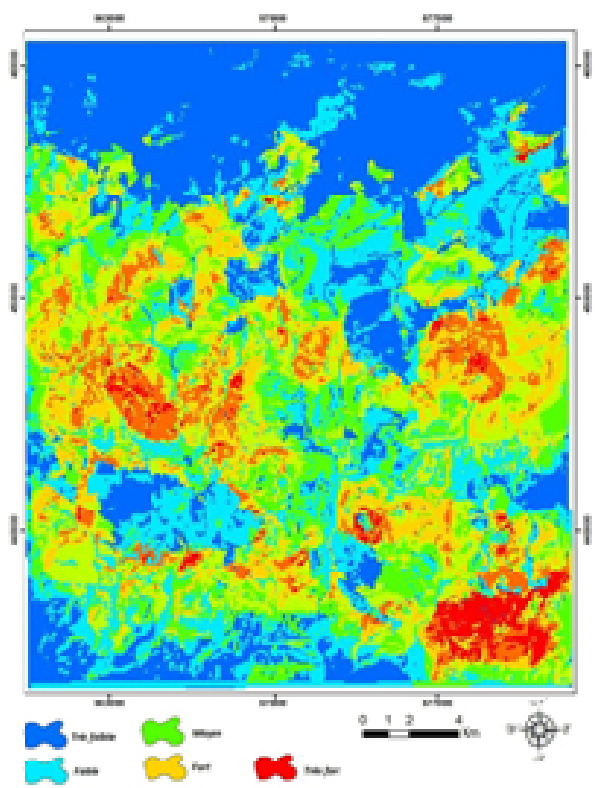

Fig.11 carte de susceptibilité aux mouvements de mass par la théorie de l'évidence de la région de Taounate_Ain Aicha

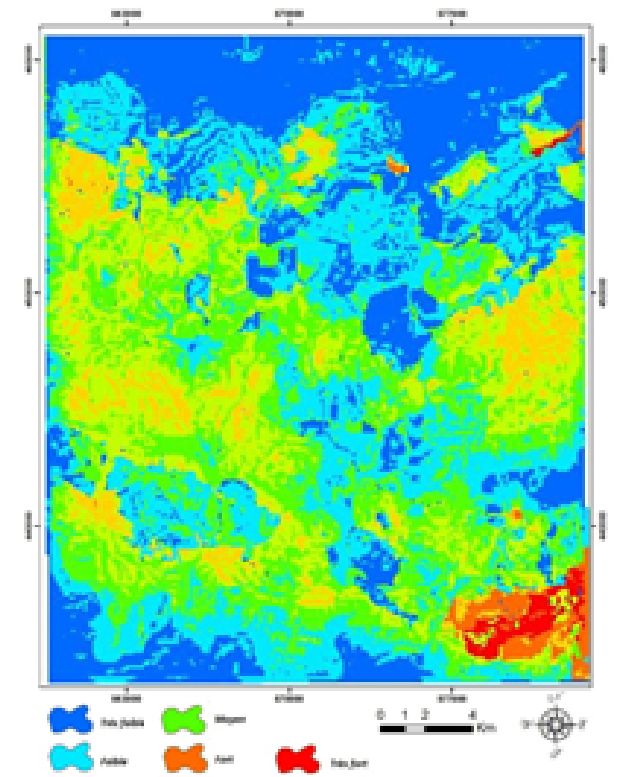

Fig.12 carte de susceptibilité aux mouvements de mass par la Régression logistique de la région de Taounate_Ain Aicha

\section{Conclusion}

Dans cet article, deux méthodes ont été utilisées pour l'analyse de la susceptibilité aux mouvements de terrain dans la région de Taounate - Ain Aicha : la théorie de l'évidence et la régression logistique. La théorie de l'évidence appliquée s'est avéré plus performante et adaptée que celle de la régression logistique à la prédiction des instabilités de terrain dans la région de Taounate - Ain Aicha. La précision de prédiction du modèle par la théorie de l'évidence est de $75 \%$ par contre celle obtenue pour la régression logistique n'est que 56\%. De plus, la carte de susceptibilité aux glissements de terrain qui en résulte montre que les zones de susceptibilité élevée à très élevée élaborée par la théorie de l'évidence présentent le pourcentage le plus élevé (62\%) de tous les glissements de terrain inventoriés par rapport aux autres zones par contre ce pourcentage n'est que $47 \%$ pour les mêmes zones sur la carte de susceptibilité obtenue par la régression logistique. La carte de susceptibilité au glissement de terrain obtenue par la théorie de l'évidence (Wof E) constitue une contribution à la gestion et à la planification territoriales urbaines et régionales dans le cadre du programme national de développement de la province de Taounate.

\section{References}

1 R. Fell, J. Corominas, C. Bonnard, L. Cascini, E. Leroi, W. Z Savage; Guidelines for Landslide Susceptibility, Hazard and Risk Zoning for Land Use Planning; On behalf of the JTC-1 Joint Technical Committee on Landslides and Engineered Slopes:, Eng. Geol., 102, 85-98, (2008). 
2 F. Guzzetti, A. Carrara, M. Cardinali, P. Reichenbach, Landslide hazardevaluation:areview of current techniques and their application in a multi-scalestudy, central Italy. Geomorphology 31, 181-216. (1999)

3 L. Ait Brahim, M. Mansour, F. Sossey Alaoui, I. EL Hamdouni, Rôle de la fracturation dans la désagrégation mécanique des calcaires et la mise en mouvement de la coulée de pierres d'Amtrasse (Rif, Maroc), Edition ISBN 9954-8407-0-2 "L'implication de la géotechnique dans le développement des infrastructures en Afrique ", 69-79 (2003).

4 D Spiegelhater., R.P. Kill-Jones, Statistical and Knowledge Approaches to Clinical Decisionsupport Systems, with an Application in Gastroenterology, Journal of the Royal Statistical Society, vol. 147, 1, 1984 p. 35-77.

5 G.F Bonham-Carter, F.P Agterberg, D.F Wright. Weights of evidence modeling: a new approach to mapping mineral potential. Statistical Applications in the Earth Science: Geological Survey of Canada Paper, vol. 89-9, pp. 171-183. (1989)

6 D.G. Kleinbaum, Logistic Regression A SelfLearning Text. Springer-Verlag, New York. (1994) https://doi.org/10.1007/978-1-4757-4108-7

7 F.P.Agterberg, Q.Cheng; Conditional independence test for weights-of- evidence modeling, Natural Resources Research, v. 11, 4, p. 249-255. (2002)

8 S. Lee, B. Pradhan, Landslide hazard mapping at Selangor, Malaysia using frequency ratio and logistic regression models, Landslides 4, 3341(2007).

9 Y. Thiery, J-P. Malet, S. Sterlacchini, A. Puissant, O. Maquaire, Landslide susceptibility assessment by bivariate methods at large scales: Application to a complex mountainous environment, Geomorphology (2007).

10 A. Fares; Essai méthodologique de la cartographie des risques naturels liés aux mouvements de terrain. Application à l'aménagement de la ville de Taounate (Rif, Maroc). Thèse Doctorat. Université de Franche Comté, (1994).

11 C.J. Van Westen, GIS in landslide hazard zonation: a review, with examples from the Andes of Colombia, Mountain Environments and Geographic Information Systems (1994).
12 L. Aït Brahim, Tectonique cassante et état de contaraintes récentes du Maroc Nord, contibution à l'étude du risque sismo-tectonique au Maroc. Thèse de doctorat d'état, Université Mohamed V, Faculté des Sciences, Rabat. (1991) 